\title{
Correction: Barik et al., LRP4 Is Critical for Neuromuscular Junction Maintenance
}

In the article "LRP4 Is Critical for Neuromuscular Junction Maintenance" by Arnab Barik, Yisheng Lu, Anupama Sathyamurthy, Andrew Bowman, Chengyong Shen, Lei Li, Wen-cheng Xiong, and Lin Mei, which appeared on pages 13892-13905 of the October 15, 2014 issue, the authors regret that the labels of the two curves were accidentally swapped in Figure 4G. This correction does not affect the interpretations or conclusions of the study. The corrected Figure 4 is shown below.

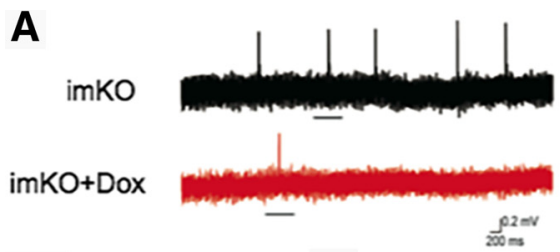

B

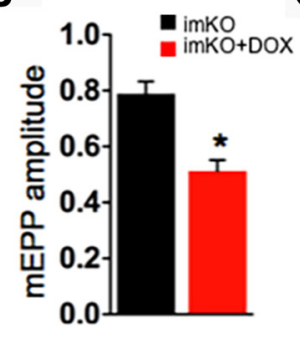

E

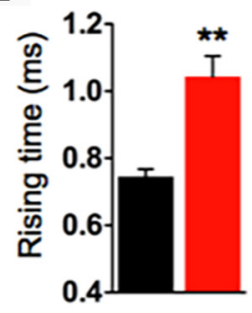

C

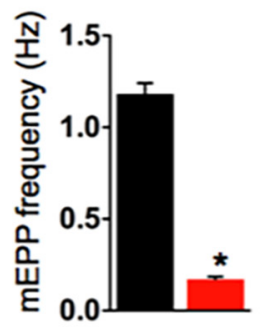

$\mathbf{F}$

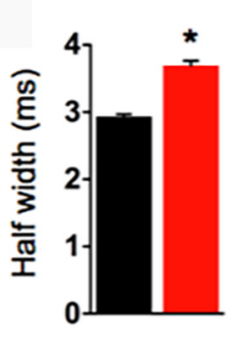

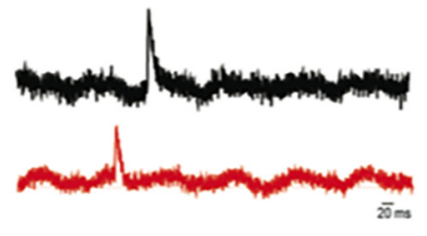

D

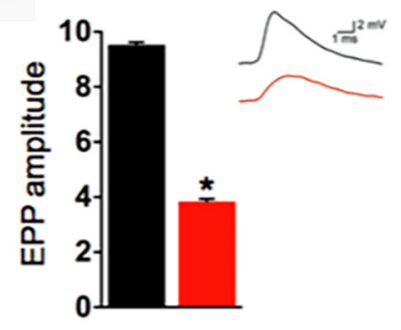

G

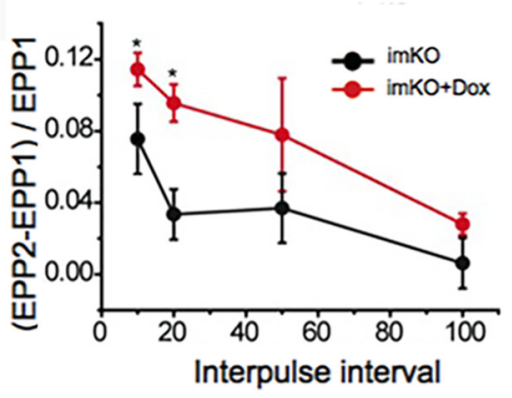

Figure 4.

\section{Correction: French et al., Dual Mechanism for Bitter Avoidance in Drosophila}

In the article "Dual Mechanism for Bitter Avoidance in Drosophila" by Alice Sarah French, Marie-Jeanne Sellier, Ali Agha Moutaz, Alexandra Guigue, Marie-Ange Chabaud, Pablo D. Reeb, Aniruddha Mitra, Yves Grau, Laurent Soustelle, and Frédéric Marion-Poll, which appeared on pages 3990-4004 of the March 4, 2015 issue, the third author's first and last names were accidentally reversed at publication. The corrected author line is: Alice Sarah French, Marie-Jeanne Sellier, Moutaz Ali Agha, Alexandra Guigue, Marie-Ange Chabaud, Pablo D. Reeb, Aniruddha Mitra, Yves Grau, Laurent Soustelle, and Frédéric Marion-Poll, which has been corrected on the online PDF version. 